\title{
Modeling Discrete Channels Based on Gilbert Model using MATLAB Software
}

\author{
Rakhimov Temurbek Omonboyevich, Ismailov Shavkat Kuzievich, Matyokubov Utkir \\ Karimovich, Eschanov Umar Kutlimuratovich, Kuchkarov Voxid Alisherovich
}

\begin{abstract}
Examining the state of discrete channels requires the study of the physical and mathematical characteristics of these channels. The Gilbert model allows for the determination of the channel state for detecting and correcting errors mainly in discrete channels. In the Gilbert model, the channel can be in two cases. It is a good case that there are no errors, and in the worst case, an error with $\mathrm{px}$ is likely to occur. The need for error-free channels for discrete channels, as well as the use of discrete channels for data transmission, as well as the use of Virtual programs for error detection and correction. The article deals with the use of Gilbert's model to detect and correct errors in discrete channels, and to combat noise in providing reliability and resilience of information. In addition, Gilbert's model is based on the modeling of the discrete channels using the MATLAB software and the removal of detected errors by a virtual model.
\end{abstract}

Keywords: information, message, channel, signal, system, model, model memory.

\section{INTRODUCTION}

Increasing the efficiency of computer systems significantly increases the amount of information stored and transmitted. The need to avoid errors, as well as the use of discrete channels in data transmission, as well as errors detection and correction programs. A set of devices that share information from one point of the data transmission system to another, provides the concept of a communication channel.

Revised Manuscript Received on December 30, 2019.

* Correspondence Author

Rakhimov Temurbek Omonboyevich, Lecturer, Department of Telecommunications Engineering, Urgench branch of Tashkent University of Information Technologies named after Muhammad al-Khwarizmi. E-mail: Rahimov_timur@bk.ru

Ismailov Shavkat Kuzievich, Head, Department of Telecommunication Engineering, Urgench branch of Tashkent University of Information Technologies named after Muhammad al-Khwarizmi.

E-mail: shavkat6819@mail.ru

Matyokubov Utkir Karimovich, Lecturer, Department of Telecommunications Engineering, Urgench branch of Tashkent University of Information Technologies named after Muhammad al-Khwarizmi.

E-mail: otkir_matyobov89@mail.ru

Eschanov Umar Kutlimuratovich, Assistant, Department of Software Engineering, Urgench Branch of Tashkent University of Information Technologies named after Muhammad al-Khwarizmi.

E-mail: bek28@list.ru

Kuchkarov Voxid Alisherovich, Assistant, Department of Telecommunications Engineering, Urgench Branch of Tashkent University of Information Technologies named after Muhammad al-Khwarizmi.

E-mail: voxidkuchkarov@umail.uz

(C) The Authors. Published by Blue Eyes Intelligence Engineering and Sciences Publication (BEIESP). This is an open access article under the CC BY-NC-ND license (http://creativecommons.org/licenses/by-nc-nd/4.0/)

\section{DISCRETE CHANNEL}

\section{A. Discrete channel}

Author (s) can send paper in the given email address of the journal. There are two email address. It is compulsory to send paper in both email address.

If discrete signals are transmitted along a channel, the channel is called a discrete channel. Discrete channels are of two types: memorable and memorable.

An unrecognizable discrete channel - the probability of the character that appears at this output depends only on the āi character.

The discrete discrete channel - the probability of the character that appears at this output depends not only on the ài character, but also on the previous entry - the icon.

\section{B. Semantics}

One of the main problems in studying these channels is to create the correct semantics. In this case, you can fight the noise and use Gilbert's model to solve the problem. Discrete channels are the basis of the digital communication system and their quality indicators depend on the requirements or needs. In the discrete channels, the Gilbert model is the main problem to verify the completeness of the semantics for a particular calculation, if calculations are created for a specific semantics, the issue of reliability is also relevant - that is, confirmed formulas but not everything that exists on the contrary, in the semantics, is confirmed in this calculation.

\section{Modeling and mathematical results}

The simplest way to learn the discrete channel model - the likelihood of errors in the Gilbert model, the unimaginable symmetric discrete channel that is subject to binomial distribution:

$$
P(k, n)=C_{n}^{k} \cdot p^{k} \cdot(1-p)^{n-k}
$$

where: $\mathrm{n}$ - the number of accepted characters;

$\mathrm{k}$ - number of bits which can be n length;

p - probability of one character error.

Taking into account the above, the likelihood of an error in the block length will be:

$$
P(\geq 1, m)=1-(1-p)^{m},
$$

and the likelihood of the blocks to be taken correctly is:

$$
P(0, m)=(1-p)^{m}
$$


Based on the above statements, using the table 1, baseline data can be modeled using the MatLab model based on the Gilbert model. It is necessary to create a binomial model for the distribution of errors in the binary symmetric channel when the probability of $\mathrm{p} 1$ and p2 errors in the blocks in the MatLab-Stateflow library. It is necessary to compare the results obtained from the modeling with the results of mathematical calculations.

For example: on the basis of the task given in the quail

Table 1.Comparing of modeling and mathemathical results

\begin{tabular}{|c|c|c|}
\hline $\mathrm{n}$ & $\begin{array}{c}\mathrm{p}_{1-\text { probability of one }} \\
\text { character error. }\end{array}$ & $\begin{array}{c}\mathrm{p}_{2-} \text { probability of one } \\
\text { character error. }\end{array}$ \\
\hline 1 & 0,01 & 0,53 \\
\hline 2 & 0,02 & 0,23 \\
\hline 3 & 0,01 & 0,45 \\
\hline 4 & 0,02 & 0,55 \\
\hline 5 & 0,03 & 0,15 \\
\hline
\end{tabular}

- Calculate the distribution of errors in the channel;

- modeling scheme in Matlab-Simulink;

- Creating a case diagram in Matlab-Stateflow;

- Detection of errors based on model;

\section{Stateflow Function In Matlab}

Based on this, in Matlab-Stateflow, Simulink will be used to create a model and placed the Chart block in the Stateflow block library into the new window window (Figure 1, marker 1).

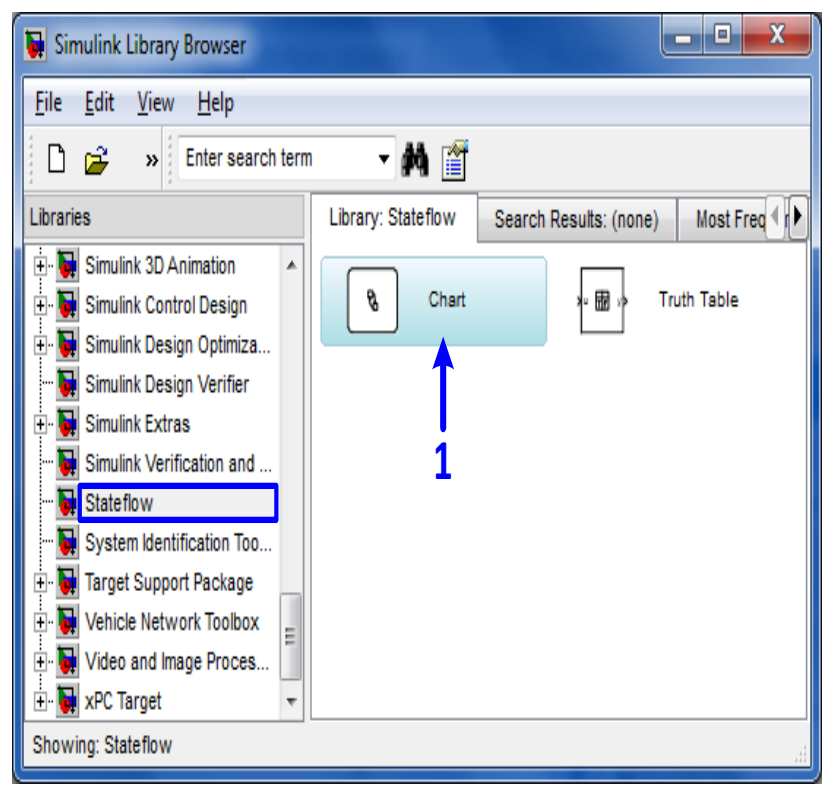

Figure 1. The "Chart" block in the Stateflow Block Library.

The working model Stateflow diagrams in the window to make sure that the block of the "Chart" apply. Stateflow diagrams are created using the Elements panel on the left side of the window that opens. Stateflow diagrams are derived from the "State" button, which indicates the state of the image (Figure 2).

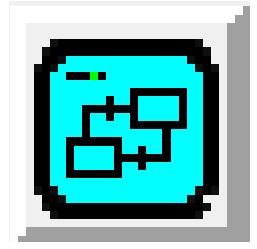

Figure 2. "State" button.

Stateflow diagrams have their own name and function so the following keywords can be used:

- entry - input parameters that indicate the movement of the current profile are entered;

- during - intermediate parameters and symbols, indicating the merging of the existing state with the following states;

- exit - Exit parameters, indicating the motion of the current state.

Stateflow diagrams indicate the initial state using the "Default Transition" button to indicate the initial state, and then set on the profile wall (Figure 3).

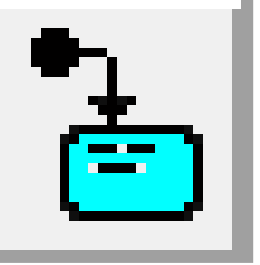

Figure 3. "Default Transition" button.

Stateflow diagrams sometimes require a combination of situations when the transition to situations, when the route is merged using the Connective Junction button (Figure 4).

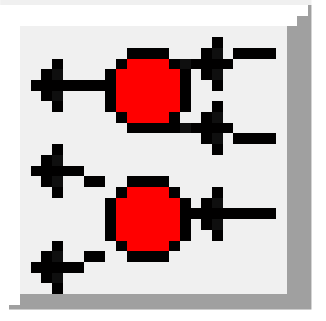

Figure 4. "Connective Junction" button.

When you switch to the conditions, the mouse pointer indicates the direction of the route. As a result, the "?" Sign will appear in the direction indicator, followed by the cursor at the "?" Icon, and the specified condition will be entered in the square bracket.

\section{RESULT}

Stateflow diagrams for binomial binary symmetric channel model are generated using the above-mentioned cases and buttons (Fig. 5). Stateflow diagrams are shown as an example where a two-bit message is transmitted along a binary symmetric channel. Here are the possible states of the two-channel message on the channel:

- there is no error message (ERRCOUNT_0);

- one bug in message (ERRCOUNT_1 mode);

- Both bits in the error message (ERRCOUNT_2 status). 


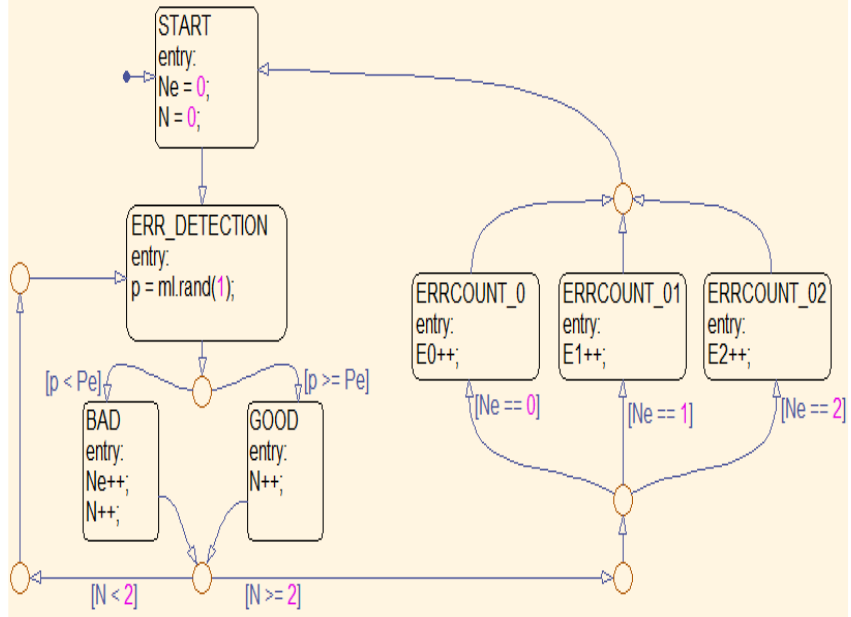

Figure 5. Stateflow diagrams for binomial binary symmetric channel model.

When the model is started, the message shown in Figure 6 must be displayed and the list below contains the list of options available on the model. This message will not appear if the parameters are already set.

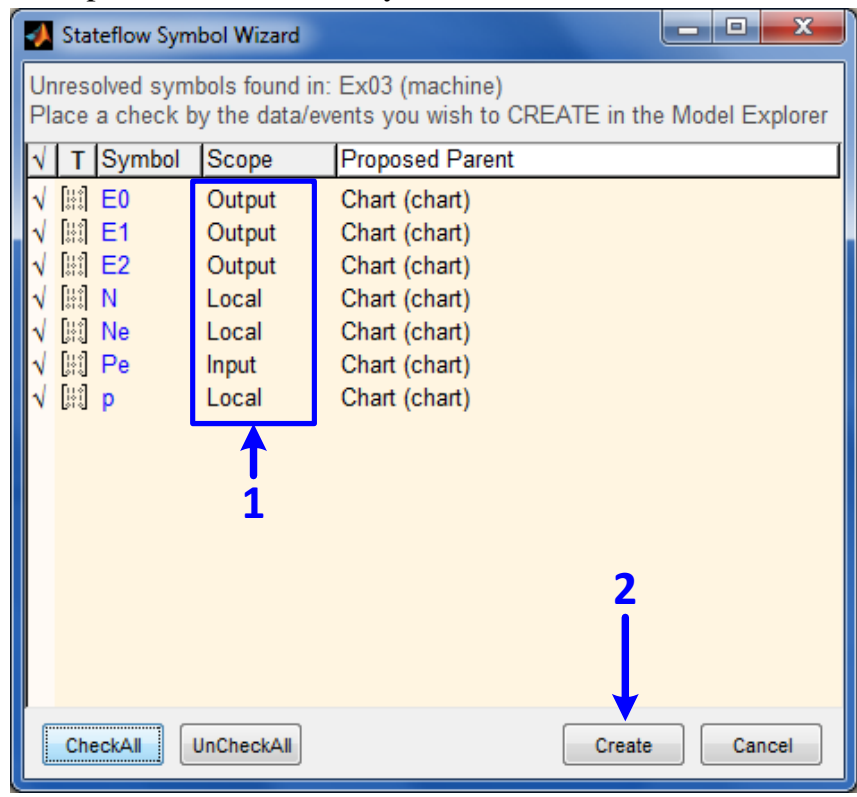

Figure 6. Variable publishing window for Stateflow diagrams.

All parameters used in the popup window need to be set. In this study, there is the following change space:

local - variable local variables only in Stateflow diagrams; input - only variables for accessing Stateflow diagrams; output - only variables that flow out of Stateflow diagrams.

Once the parameters have been changed, the appearance of the "Chart" block in the Simulink model will come to mind in Figure 7.

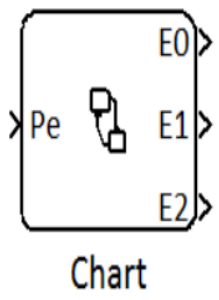

Figure 7. Views of the Chart after the Stateflow Charts have been created.

After these actions, the "Chart" block is added to the
"Display" block, and the "Constant" block is added (Figure 8).

Display and Constant blocks are obtained from the Sinks block library and the Sources block library. Display and Constant blocks are obtained from the Sinks block library and the Sources block library.

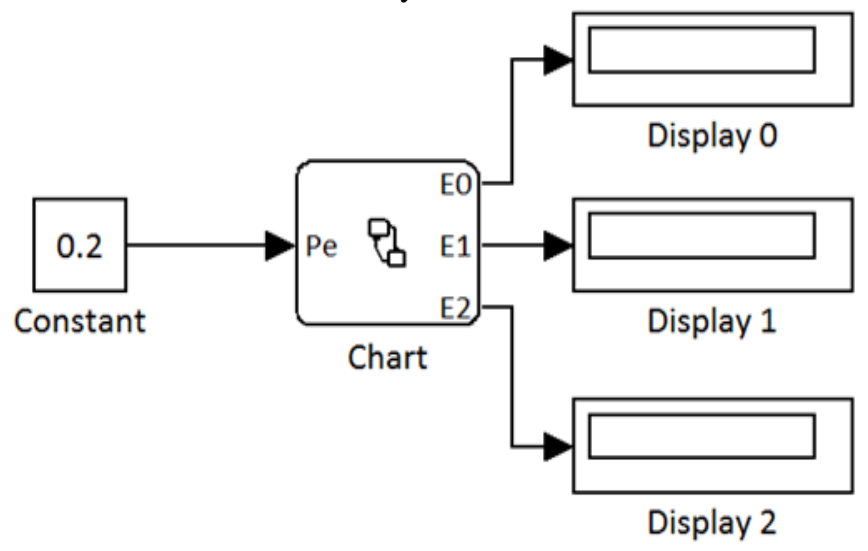

Figure 8. Binomial binary symmetrical channel diagram in Matlab-Simulink.

\section{CONCLUSION}

In summary, these studies can be used to teach experimental training on "Theory of Information and Coding", which is taught to the "Telecommunication Technologies" personalities through virtual immitation modeling. The reason for this is that students are not allowed to collect and use telecommunication devices in their home environment, and this program can easily be used in the home environment to capture the Matlab system using the standard interface elements and blocks of the input circuit interface block, can learn. In particular, "Telecommunication technologies" specialists will be able to further improve their knowledge and to further strengthen their knowledge by applying an independent $60-70 \%$ of their experiences through Matlab software.

In turn, thanks to the formation of distance learning, the modern education system is rising to a new stage of its development - the formation and development of the educational and informational environment. The student's ability to work independently with computer technology is an attempt to find a solution to the problem of the student's self-reliance and to raise awareness on information and communication technologies.

\section{REFERENCES}

1. M. Guizani, A. Rayes., "Network Modeling and Simulation".- John Wiley \& Sons Ltd, 2010-p-236.

2. Michel C.Jeruchim., "Simulation of Communication Systems". - New York, Kluwer Academic Publishers, 2002-p-526.

3. K.Wehrle, M.Gunes Modeling and Tools for Network Simulation.Springer-Verlog Berlin Heidelberg, 2010.

4. Jack Keil Wolf, Simulation of Communication Systems. - New York, Kluwer Academic Publishers, 2000-p-561.

5. A. J. Viterbi, Principles of Coherent Communication, McGraw-Hill, New York (1966).

6. A. M. Law and W. D. Kelton, Simulation Modeling and Analysis, 3rd Edition, McGraw Hill, 2000 
7. M. Guizani and A. Rayes, Designing ATM Switching Networks, McGraw Hill, 1997.

8. Rakhimov Temurbek, "Matlab-simevents are modeling a camergus servicing system for moving it information" Scientific-technical journal Turin polytechnic university in Tashkent., Tashkent $-2019 .$, p-71

\section{AUTHORS PROFILE}

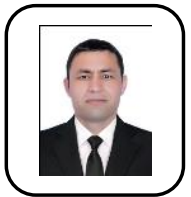

Rakhimov Temurbek Omonboyevich, Lecturer, Department of Telecommunications Engineering, Urgench branch of Tashkent University of Information Technologies named after Muhammad al-Khwarizmi. Email adress: Rahimov_timur@bk.ru

Telephone number: +99894-637-3787

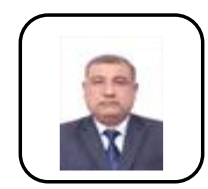

Ismailov Shavkat Kuzievich, Head of the Department of Telecommunication Engineering of Urgench branch of Tashkent University of Information Technologies named after Muhammad al-Khwarizmi.

Email adress: shavkat6819@mail.ru

Telephone number: +99899-961-75-24

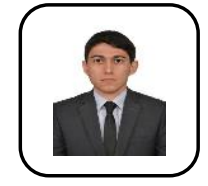

Matyokubov Utkir Karimovich, Lecturer, Department of Telecommunications Engineering, Urgench branch of Tashkent University of Information Technologies named after Muhammad al-Khwarizmi.

Email adress: otkir_matyobov89@mail.ru

Telephone number: +99899-968-80-06

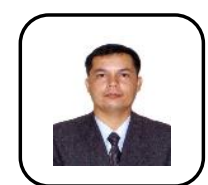

Eschanov Umar Kutlimuratovich, Assistant, Department of Software Engineering, Urgench Branch of Tashkent University of Information Technologies named after Muhammad al-Khwarizmi.

Email adress: bek28@list.ru

Telephone number: +99897-457-76-27

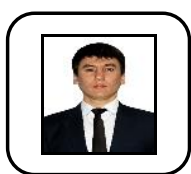

Kuchkarov Voxid Alisherovich, Assistant, Department of Telecommunications Engineering, Urgench Branch of Tashkent University of Information Technologies named after Muhammad al-Khwarizmi.

Email adress: voxidkuchkarov@umail.uz

Telephone number: +99897-452-76-27 